\title{
Serological diagnosis of Chagas disease: evaluation and characterisation of a low cost antigen with high sensitivity and specificity
}

\author{
Yelitza Campos¹, Luis Briceño', Kate Reina1, Katherine Figarella², José Luis Pérez¹, Walter Mosca ${ }^{+} / 1$ \\ ${ }^{1}$ Laboratorio de Fisiopatología (Nodo I LANPIP), Instituto de Biomedicina, Facultad de Medicina, Universidad Central de Venezuela, \\ San Nicolás a Providencia, Sector Hospital Vargas, San José 1010, Caracas, Venezuela Fundación Instituto de Estudios Avanzados, \\ Centro de Biotecnología, Caracas, Venezuela
}

In spite of evident progress in the serology of Chagas disease, the requirement for new diagnostic antigens persists. We have evaluated different antigens obtained from Trypanosoma cruzi grown in medium rich in nutrients or under nutrient stress, autoclaved or sonicated and fractionated by differential centrifugation. The resulting antigens were evaluated for diagnosis of Chagas disease using ELISA. Immunofluorescence of the parasites demonstrated that nutrient stress induced changes in the distribution and density of antigens recognised by a pool of sera from experimentally infected mice. When evaluated using ELISA, it was evident that most fractions had good sensitivity but poor specificity. Surprisingly, the best specificity and sensitivity was observed with parasites cultured under nutrient stress and autoclaved. Furthermore this antigen had low cross reactivity with sera from other parasitic diseases, Leishmaniasis in particular. Western blot analysis demonstrated that autoclaving seems to non-specifically eliminate cross-reactive antigens. In conclusion, autoclaving epimastigotes of T. cruzi, after nutrient stress, allowed us to obtain an antigen that could be used in the serological diagnosis of Chagas disease.

Key words: Chagas disease - diagnose - Trypanosoma cruzi - antigens

Diagnosis of Chagas disease in patients chronically infected with Trypanosoma cruzi is generally based on the detection of specific antibodies against antigens from epimastigotes of $T$. cruzi. Different diagnostic techniques and different antigens have been used to detect these specific antibodies. Because of differences in sensitivity and/or specificity, there is controversy about which antigen is the most efficient (Berrizbeitia et al. 2006, Zarate-Blades et al. 2007). In this context, the availability of an antigen with high sensitivity and specificity, low cross reactivity and high stability and that can be produced at a low cost should facilitate the implementation of at least two different serological tests for the diagnosis of Chagas disease as recommended by the World Health Organization (Saez-Alquezar et al. 2003, Schmunis 2007).

In previous studies, we observed that parameters like culture conditions, inhibition of proteolytic activity and processing methods significantly affect the sensitivity and specificity of the antigens (unpublished observations). Based on those studies, we have evaluated the antigenicity of different complex antigens obtained with different culture conditions (with and without nutrient stress) and different methods of processing the parasites (sonication or autoclaving).

A total of 243 serum specimens from healthy blood bank donors with negative serology for Chagas disease

Financial support: FONACIT (LAB G2000001530), LANPIP (LAB2000001639), CDCH (PI 09-35-5286-2007), LOCTI (P935015) + Corresponding author:wmosca@gmail.com

Received 13 February 2009

Accepted 20 August 2009 $(n=14)$, Chagas disease $(n=32)$ confirmed by three different serological tests, cutaneous leishmaniasis $(n=58)$, visceral leishmaniasis $(n=87)$, malaria $(n=15)$, schistosomiasis $(n=23)$ and toxoplasmosis $(n=16)$ were used, at dilutions of 1:500, in the present study. The procedures and sample utilisation methods were approved by the Ethical Committee for Human Research at the Instituto de Biomedicina, Universidad Central de Venezuela.

T. cruzi, strain Y, was grown at $25^{\circ} \mathrm{C}$ in a modified LIT medium formulated as follows: $4.0 \mathrm{~g}$ of $\mathrm{NaCl}, 0.4$ $\mathrm{g}$ of $\mathrm{KCl}, 8.0 \mathrm{~g}$ of $\mathrm{Na}_{2} \mathrm{HPO}_{4}, 5.0 \mathrm{~g}$ of tryptose, $5.0 \mathrm{~g}$ of liver extract and $2.5 \mathrm{~g}$ of yeast extract were combined in a final volume of $500 \mathrm{~mL}$ at $\mathrm{pH} 7.2$, autoclaved, adjusted to $1 \mathrm{~L}$ with sterile DMEM (GIBCO BRL, Life Technologies Inc, USA) and supplemented with $0.2 \%$ defibrinated rabbit blood, $2 \%$ foetal bovine serum (FBS), $0.2 \%$ dextrose and $50 \mu \mu \mathrm{g} / \mathrm{mL}$ gentamicin.

Leishmania infantum promastigotes were grown at $25^{\circ} \mathrm{C}$ in a modified LIT medium containing $15 \mathrm{~g}$ of tryptose, $2 \mathrm{~g}$ of liver extract, $5 \mathrm{~g}$ of yeast extract, $4 \mathrm{~g}$ of glucose, $9 \mathrm{~g}$ of NaCl, $0.4 \mathrm{~g}$ of $\mathrm{KCl}, 7.5 \mathrm{~g}$ of $\mathrm{Na}_{2} \mathrm{HPO}_{4}$ and 0.02 $\mathrm{g}$ of haemin in $8 \mathrm{~mL}$ of $0.1 \mathrm{~N} \mathrm{NaOH}, \mathrm{pH}^{2} 7.5$, which was diluted to a final volume of $1 \mathrm{~L}$, autoclaved and supplemented with $10 \%$ FBS.

Epimastigotes and promastigotes from the different culture conditions were processed by two methods, autoclaving and sonication. (i) for the autoclaved epimastigotes or promastigotes (AE), cultures of parasites in stationary phase were centrifuged at 2,000 $\mathrm{g}$ for $10 \mathrm{~min}$; the pellet was resuspended and washed three times in sterile phosphate buffered saline (PBS) at $\mathrm{pH}$ 7.2. After the last wash, they were resuspended with PBS at $30 \times 10^{6}$ parasites/mL and autoclaved; (ii) for the nutrient stress autoclaved epimastigotes or promastigotes (ASE), parasites in stationary phase were harvested as in (i), resus- 
pended at $30 \times 10^{6}$ parasites $/ \mathrm{mL}$ with DMEM supplemented with gentamicin $(50 \mu \mathrm{g} / \mathrm{mL})$, incubated at $25^{\circ} \mathrm{C}$ for $24 \mathrm{~h}$ and autoclaved as described for AE; (iii) for the sonicated epimastigotes or promastigotes, parasites were processed as described in (i) and (ii), but instead of being autoclaved, they were sonicated twice for $20 \mathrm{~s}$ at $4^{\circ} \mathrm{C}$ and 50 watts (Branson 250, Branson Ultrasonic Corporation, USA). With this procedure, two crude antigens were obtained: sonicated antigen of stationary phase (SE) and of nutrient stress (SSE) parasites. Both were aliquoted and stored at $-20^{\circ} \mathrm{C}$; (iv) the subcellular fractions were obtained by subjecting sonicated parasites to differential centrifugation.

Leishmania sp. antigens were identified as autoclaved or sonicated. Protein concentrations were assessed by a colourimetric assay (Micro BCA protein assay, PIERCE, USA).

The antigenic preparations of T. cruzi or Leishmania sp. were evaluated by an indirect ELISA. Optimisation of antigen, serum and conjugate concentrations was performed by checkerboard titration. Briefly, ELISA plates (Maxisorp, Nunc International Corporation) were coated with $100 \mu \mathrm{L}(2 \mu \mathrm{g} /$ well $)$ of antigen and $100 \mu \mathrm{L}$ of car- bonate buffer $\left(0.016 \mathrm{M} \mathrm{Na}_{2} \mathrm{CO}_{3}, 0.034 \mathrm{M} \mathrm{NaHCO}_{3}, \mathrm{pH}\right.$ 9.6). After overnight incubation at $4^{\circ} \mathrm{C}$, the plates were washed three times for $10 \mathrm{~min}$ with TBS-T $150(0.150 \mathrm{M}$ $\mathrm{NaCl}, 50 \mathrm{mM}$ Tris Base, $0.01 \%$ Tween 20, $\mathrm{pH}$ 8), blocked for $1 \mathrm{~h}$ at $37^{\circ} \mathrm{C}$ with TBS-T 150 supplemented with $5 \%$ low-fat dry milk and 1\% FBS and washed again. Serum samples, diluted 1:500 with TBS-T, were added (two wells for each serum sample) and incubated for $1 \mathrm{~h}$ at $37^{\circ} \mathrm{C}$. The wells were washed three times and incubated with alkaline phosphatase-conjugated goat anti-human IgG $(\mathrm{H}+\mathrm{L}$; GIBCO BRL, USA) for $1 \mathrm{~h}$. After washing the wells three times, PNPP substrate (PIERCE) in diethanolamine buffer was added to each well and incubated for $30 \mathrm{~min}$ for colour development. Optical density (OD) was measured at $405 \mathrm{~nm}$ in a Multiskan TX microplate reader (Thermo Electron Corporation). The lower limit of detection (cut-off) was determined as the mean OD of the negative control serum plus two standard deviations. Sensitivity, specificity, confidence limits, significance and graphics were calculated using the GraphPad InStat 3.02 (GraphPad software, San Diego, CA) and Epitable software (Centers for Disease Control and Prevention, Atlanta, GA).

TABLE I

Sensitivity and specificity of autoclaved (A) and sonicated (S) subcellular fraction and nutrient stress or normal culture epimastigote of Trypanosoma cruzi autoclaved or sonicated were evaluated using an ELISA assay with sera from patients with Chagas disease and healthy controls

\begin{tabular}{|c|c|c|c|c|c|c|}
\hline & Positives & Negatives & $\mathrm{Fp}$ & Fn & $\begin{array}{l}\text { Sensitivity } \\
\text { (CL95) }\end{array}$ & $\begin{array}{l}\text { Specificity } \\
\text { (CL95) }\end{array}$ \\
\hline P1000 A & 32 & 13 & 12 & 0 & $\begin{array}{c}100 \\
(89.11-100)\end{array}$ & $\begin{array}{c}7.69 \\
(0.19-36.03)\end{array}$ \\
\hline $\mathrm{P} 1000 \mathrm{~S}$ & 32 & 13 & 10 & 0 & $\begin{array}{c}100 \\
(89.11-100)\end{array}$ & $\begin{array}{c}23.08 \\
(5.04-53.81)\end{array}$ \\
\hline P5000 A & 32 & 13 & 12 & 2 & $\begin{array}{c}93.75 \\
(79.19-99.23)\end{array}$ & $\begin{array}{c}8.33 \\
(0.21-38.48)\end{array}$ \\
\hline $\mathrm{P} 5000 \mathrm{~S}$ & 32 & 13 & 9 & 1 & $\begin{array}{c}96.88 \\
(83.78-99.92)\end{array}$ & $\begin{array}{c}30.77 \\
(9.09-61.43)\end{array}$ \\
\hline P11000 A & 32 & 13 & 2 & 1 & $\begin{array}{c}96.96 \\
(83.78-99.92)\end{array}$ & $\begin{array}{c}84.62 \\
(54.55-98.08)\end{array}$ \\
\hline P11000 S & 32 & 13 & 7 & 0 & $\begin{array}{c}100 \\
(89.11-100)\end{array}$ & $\begin{array}{c}46.15 \\
(19.22-74.87)\end{array}$ \\
\hline S11000 A & 32 & 13 & 7 & 0 & $\begin{array}{c}100 \\
(89.11-100)\end{array}$ & $\begin{array}{c}46.15 \\
(19.22-74.87)\end{array}$ \\
\hline $\mathrm{S} 11000 \mathrm{~S}$ & 32 & 13 & 11 & 0 & $\begin{array}{c}100 \\
(89.11-100)\end{array}$ & $\begin{array}{c}15.38 \\
(1.92-45.45)\end{array}$ \\
\hline ASE & 30 & 14 & 0 & 0 & $\begin{array}{c}100 \\
(88.43-100)\end{array}$ & $\begin{array}{c}100 \\
(88.43-100)\end{array}$ \\
\hline SSE & 30 & 14 & 7 & 0 & $\begin{array}{c}100 \\
(88.43-100)\end{array}$ & $\begin{array}{c}50 \\
(23.04-76.96)\end{array}$ \\
\hline $\mathrm{AE}$ & 30 & 10 & 0 & 3 & $\begin{array}{c}90 \\
(73.47-97.89)\end{array}$ & $\begin{array}{c}100 \\
(69.15-100)\end{array}$ \\
\hline $\mathrm{SE}$ & 30 & 10 & 0 & 1 & $\begin{array}{c}96.6 \\
(82.78-99.92)\end{array}$ & $\begin{array}{c}100 \\
(69.15-100)\end{array}$ \\
\hline
\end{tabular}

AE: autoclaved epimastigotes; ASE: autoclaved nutrient stress epimastigotes; CL: confidence limits; Fn: false negative; Fp: false positive; P1000 A: pellet 1000g autoclaved; P1000 S: pellet 1000g sonicated; P5000 A: pellet 5000g autoclaved; P5000 S: pellet $5000 \mathrm{~g}$ sonicated; P11000 A: pellet $11000 \mathrm{~g}$ autoclaved; P11000 S: pellet $11000 \mathrm{~g}$ sonicated; S11000 A: supernatant $11000 \mathrm{~g}$ autoclaved; S11000 S: supernatant 11000g sonicated; SE: sonicated epimastigotes; SSE: sonicated nutrient stress epimastigotes. 
As shown in Table I, no significant difference could be established among the sonicated and autoclaved antigens and ASE had the best combination of sensitivity (100\%) and specificity $(100 \%)$ (Table I). All subcellular fractions had good sensitivity, but, independent of the processing method used, their specificity was lower than ASE. Consequently, it seems that a complex antigenic mixture is required to obtain the best specificity and sensitivity.

Interestingly, when the parasites were sonicated, nutrient stress did not improve antigen performance, as shown by a high number of false positives. This suggests that the antigens that react non-specifically with antibodies are preferentially denatured when nutrientstressed epimastigotes are autoclaved. Our data show that ASE has a high sensitivity and specificity and the cost to produce it is low. Furthermore, the agreement of the data obtained with unfractionated antigens with the expected values (ASE 100; SE 97.5; AE 92.5; SSE 84.09) indicates that ASE could potentially be used for routine diagnosis of Chagas disease.

The fact that autoclaving an antigen improves its specificity without affecting its sensitivity was unexpected. To evaluate the validity of this observation for other species, we assessed the effect of autoclaving Leishmania sp. promastigotes. The data obtained (sensitivity $=0 \%$ ) show that, in spite of the close phylogenetic relationship between T. cruzi and Leishmania, the antigenicity of promastigotes was severely affected by autoclaving.

Cross reactivity with cutaneous or visceral leishmaniasis frequently limits the specificity of serological tests for the diagnosis of Chagas disease. When analysing the data summarised in Table II, it is evident that the percentage of patients with confirmed leishmaniasis and positive serology for Chagas using ASE was low (less than $10 \%$ ). In Venezuela, there is significant overlap of the areas where leishmaniasis and Chagas disease are endemic and it is difficult to exclude a previous infection with Leishmania; however, when sera from patients with leishmaniasis that tested positive with ASE were evaluated with a commercial kit (BIOSChile), all were positive for Chagas disease. Consequently, we can conclude that the specificity of our antigen preparation is as good as the specificity of commercial antigens.

Contrary to what was expected, autoclaving epimastigotes of T. cruzi did not affect antigen sensitivity and specificity and decreased the cross reactivity observed with sera from other parasitic diseases.

To more precisely evaluate the consequences of autoclaving T. cruzi epimastigotes, we performed Western blots (Towbin et al. 1979) on the SE and ASE epimastigotes using sera from patients with Chagas disease $(\mathrm{n}=10)$ and visceral leishmaniasis $(\mathrm{n}=5)$ and pooled sera from patients with Chagas disease. Sera from patients with Chagas disease revealed more peptides of low and high molecular weights in ASE (Fig. 1). As expected, due to the denaturing effect of autoclaving, most bands identified by Western blot of ASE were of low molecular weight. However, when we compared the bands that reacted with sera from patients with visceral leishmaniasis (Fig. 1, Lanes 11-15), it was evident that bands observed in the Western blot of $\mathrm{AE}(26,37$ and $49 \mathrm{kDa})$ are not present in the Western blot of ASE. The principal source of the false positive serological diagnosis of Chagas disease is cross reactivity with sera from patients with Leishmaniasis. Our findings suggest that cross-reactive antigens of $T$. cruzi are more susceptible to autoclaving than specific antigens. Although the reactivity with a band of $26 \mathrm{kDa}$ was lost after autoclaving with sera of visceral leishmaniasis patients, reactivity persisted with sera of Chagasic patients. This suggests the presence of at least two 26-kDa polypeptides, one that is responsible for the cross reactivity with visceral leishmaniasis sera

\section{TABLE II}

Evaluation of the cross reactivity of autoclaved nutrient stress epimastigotes with an ELISA assay and sera from patients with cutaneous leishmaniasis, visceral leishmaniasis, malaria, schistosomiasis and toxoplasmosis

\begin{tabular}{lccc}
\hline & & & $\begin{array}{c}\text { Specificity } \\
(\%)\end{array}$ \\
\hline Cutaneous leishmaniasis & 56 & 5 & 91 \\
Visceral leishmaniasis & 87 & 8 & 90.8 \\
Toxoplasmosis & 16 & 0 & 100 \\
Malaria & 15 & 0 & 100 \\
Esquistosomiasis & 23 & 1 & 95.65 \\
\hline
\end{tabular}

Fp: false positive.
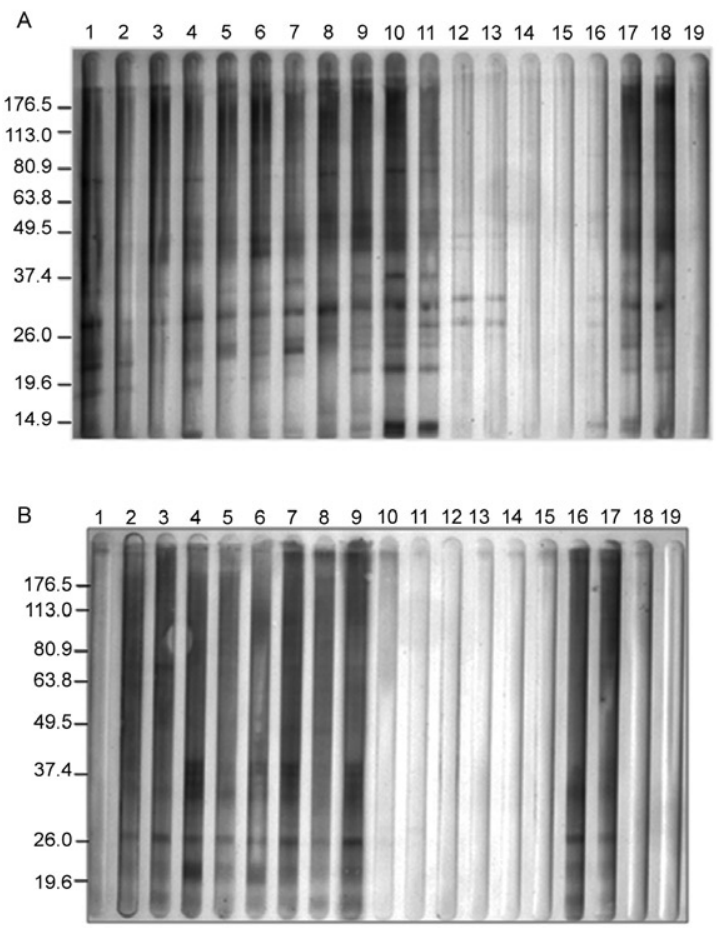

Fig. 1: Western blot of Trypanosoma cruzi antigen. A: sonicated epimastigotes; B: autoclaved, nutrient stress epimastigotes. Lines 1-10: chagasic patients; 11-15: leishmaniasis patients; 16-18: pool of serum from chagasic patients [A: 16 (low titre), 17 (intermediate titre), 18 (high titre); B: 16 (intermediate titre), 17 (high titre), 18 (low titre)]; 19: pool of serum from healthy individuals. The presence of bound antibodies was detected with BCIP/NBT (Gibco Laboratories, Grand Island, NY, USA) and analyzes using a Gel Doc System (BIO-RAD, USA). 


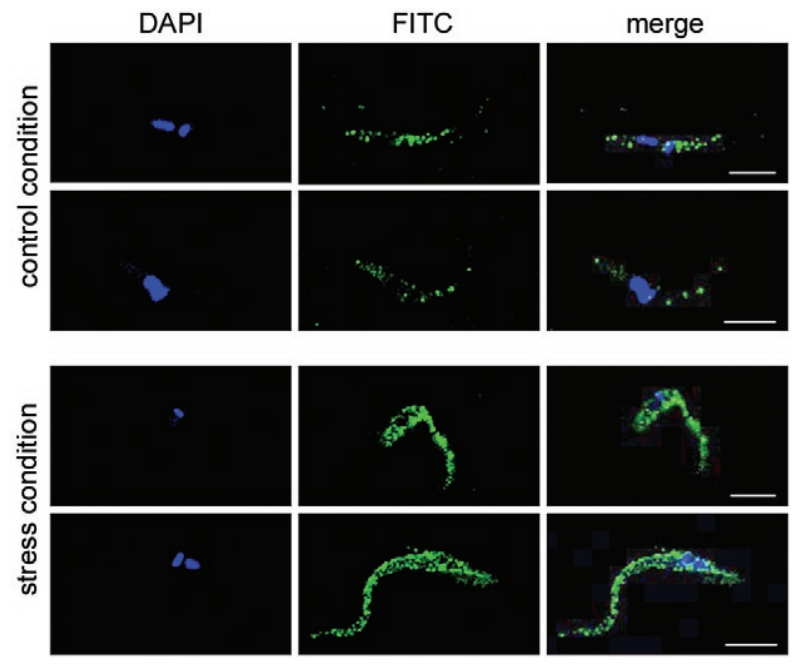

Fig. 2: immunofluorescence of epimastigotes of Trypanosoma cruzi with a pool of serum from chronic infected mouse. Upper panel: control epimastigotes of T. cruzi cultivated in complete medium (control condition); lower panel: epimastigotes of $T$. cruzi subjected to nutrient stress (stress condition). Nucleus and kinetoplast were stained using DAPI. Secondary antibody: FITC labeled antimouse IgG. Bar in merge images represents $5 \mu \mathrm{m}$.

and one that is specific for Chagas disease. Morgado et al. (1989) described two bands (46 and $25 \mathrm{kDa}$ ) specific to Chagas disease and both bands are present in SE. After autoclaving, the $46-\mathrm{kDa}$ band disappeared, but the $25-\mathrm{kDa}$ band persisted and seemed to be important for the specificity and sensitivity of ASE.

To document the effect of nutrient stress in living parasites, $1 \times 10^{6}$ epimastigotes from cultures in stationary phase with and without nutrient stress for $24 \mathrm{~h}$ were fixed overnight at $4^{\circ} \mathrm{C}$ with $4 \%$ paraformaldehyde in PBS. After fixation, the cells were washed twice with PBS and incubated in glycine PBS $\left(50 \mathrm{mM} \mathrm{Na}_{2} \mathrm{PO}_{4}, 50\right.$ $\mathrm{mM}$ glycine, $\mathrm{pH}$ 7.2) for $15 \mathrm{~min}$. Antiserum of an infected mouse was diluted, according to previous standardisation, 1:50 in 1\% bovine serum albumin (BSA) in PBS and cells were incubated with antiserum for $1 \mathrm{~h}$ at $4^{\circ} \mathrm{C}$. The primary antibody was removed by washing three times with $1 \%$ BSA in PBS followed by incubation with FITC rabbit anti mouse IgG $(\mathrm{H}+\mathrm{L}$; DAKO) at 1:500 in $1 \%$ BSA for $1 \mathrm{~h}$. Finally, cell nuclei were stained with DAPI (0.1 mg mL $\mathrm{m}^{-1}$ final concentration; Fluka) for $5 \mathrm{~min}$, followed by washing three times with $1 \%$ BSA and once with sterile water. Parasites were transferred to slides, mounted in Fluoromount-G (Southern-Biotech) and examined with a 100X oil immersion objective lens in a $\mathrm{C} 1$ Confocal Microscope (Nikon). Images were obtained using the EZ-C1 software (Nikon). When we compared the control fluorescence pattern to the nutrient-stressed epimastigotes pattern, the patterns differed in intensity as well as in distribution (Fig. 2). Control parasites had few small discrete spots in the posterior end, the cell body and the tip of the flagellum. This labelling was clearly different from the larger and more abundant fluorescent spots observed in the nutrient-stressed epimastigotes.
This suggests that nutrient stress increased the density of antigens recognised by the antiserum and/or induced the expression of different antigenic molecules in the parasite. The change in the pattern of labelling is associated with improvements in sensitivity and specificity of the antigens obtained from epimastigotes subjected to nutrient stress and autoclaving. As reported by other investigators, modifications in culture conditions, like those evaluated here, induce biochemical and molecular changes in the antigens produced (Contreras et al. 1994, 1998). The expression of these changes is evident in the immunofluorescence of epimastigotes subjected to different culture conditions.

Our data suggest that somehow nutrient stress and autoclaving, in a manner that seems to be specific to epimastigotes, induce preferential loss of cross-reactive antigens while maintaining enough specific antigens for Chagas disease.

In conclusion, the low cross reactivity, high sensitivity and specificity of ASE makes it suitable for the immunodiagnosis of Chagas disease.

\section{ACKNOWLEDGEMENTS}

To Dr. Oscar Noya, from Instituto de Medicina Tropical, Universidad Central de Venezuela, and Dra. Mariam Ulrich, from Instituto de Biomedicina, Universidad Central de Venezuela, for providing serum samples, to Dr. Enrique Arciniegas, for providing secondary antibody and his assistance with immunofluorescence, and to Dr. Rafael Borges, for his assistance for the statistical analysis.

\section{REFERENCES}

Berrizbeitia M, Ndao M, Bubis J, Gottschalk M, Ache A, Lacouture S, Medina M, Ward B J 2006. Field evaluation of four novel enzyme immunoassays for Chagas' disease in Venezuela blood banks: comparison of assays using fixed-epimastigotes, fixedtrypomastigotes or trypomastigote excreted-secreted antigens from two Trypanosoma cruzi strains. Transfus Med 16: 419-431.

Contreras VT, Araque W, Delgado VS 1994. Trypanosoma cruzi: metacyclogenesis in vitro--I. Changes in the properties of metacyclic trypomastigotes maintained in the laboratory by different methods. Mem Inst Oswaldo Cruz 89: 253-259.

Contreras VT, De Lima AR, Zorrilla G 1998. Trypanosoma cruzi: maintenance in culture modify gene and antigenic expression of metacyclic trypomastigotes. Mem Inst Oswaldo Cruz 93: 753-760.

Morgado MG, Ivo-dos-Santos J, Pinho RT, Argüelles E, Rezende JM, Galvão-Castro B 1989. Trypanosoma cruzi: identification of specific epimastigote antigens by human immune sera. Mem Inst Oswaldo Cruz 84: 309-314.

Saez-Alquezar A, Murta M, Pereira Marques W, Rodrigues da Silva G 2003. The results of an external quality control program for serological screening for antibodies against Trypanosoma cruzi in blood donors in Brazil. Rev Panam Salud Publica 13: 129-137.

Schmunis GA 2007. Epidemiology of Chagas disease in non endemic countries: the role of international migration. Mem Inst Oswaldo Cruz 102: 75-86.

Towbin H, Staehelin T, Gordon J 1979. Electrophoretic transfer of proteins from polyacrylamide gels to nitrocellulose sheets: procedure and some applications. Proc Natl Acad Sci USA 76: 4350-4354.

Zarate-Blades CR, Blades N, Nascimento MS, da Silveira JF, Umezawa ES 2007. Diagnostic performance of tests based on Trypanosoma cruzi excreted-secreted antigens in an endemic area for Chagas' disease in Bolivia. Diagn Microbiol Infect Dis 57: 229-232. 